\title{
A New Low-energy Plastic Scintillation Neutron Detector For Real-time Pulse Shape Discrimination
}

\section{Stuhl*}

Center for Nuclear Study, University of Tokyo, 2-1 Hirosawa, Wako, Saitama, Japan

RIKEN Nishina Center, 2-1 Hirosawa, Wako, Saitama, Japan

E-mail: stuhl@ens.s.u-tokyo.ac.jp

\section{Sasano}

RIKEN Nishina Center, 2-1 Hirosawa, Wako, Saitama, Japan

\section{K. Yako}

Center for Nuclear Study, University of Tokyo, 2-1 Hirosawa, Wako, Saitama, Japan

\section{J. Yasuda}

Department of Physics, Kyushu University, Motooka 744, Fukuoka, Nishi, Japan

\section{H. Baba}

RIKEN Nishina Center, 2-1 Hirosawa, Wako, Saitama, Japan

\section{S. Ota}

Center for Nuclear Study, University of Tokyo, 2-1 Hirosawa, Wako, Saitama, Japan

\section{T. Uesaka}

RIKEN Nishina Center, 2-1 Hirosawa, Wako, Saitama, Japan

\begin{abstract}
A new neutron detector has been designed to detect low-energy neutrons in $(p, n)$ chargeexchange reactions in inverse kinematics. Our detector based on EJ-299-34 plastic scintillator and coupled to digital readout system which provides opportunity for pulse shape discrimination (PSD) of the detected particles as well as intelligent triggering based on PSD. This device enables one to reduce the gamma-ray background by one order of magnitude. We plan to use it in our experiments to study the spin-isospin responses of nuclei along the neutron drip line. The new system can be used, however, for any reaction study that involves emission of low-energy neutrons $(100 \mathrm{keV}-1 \mathrm{MeV})$ where the background suppression and the increased signal-to-noise ratio are crucial.
\end{abstract}

The 26th International Nuclear Physics Conference

11-16 September, 2016

Adelaide, Australia

${ }^{*}$ Speaker. 


\section{Introduction}

In the last decades, beams of exotic radioactive isotopes with various properties became available at new facilities (RIKEN, GANIL, MSU-NSCL, GSI/FAIR). This enlarging of the scientific field, lead to study many static properties of exotic nuclei, such as, mass, deformation, singleparticle-occupancy of nuclear orbits. On the other hand, the researches of dynamic properties, which manifest themselves in higher excitation energies, are not investigated. The spin-isospin responses of very exotic nuclei, i.e., nuclei along the neutron drip line, have not yet been studied. Until now, the spin-isospin collectivity was investigated only in stable isotopes [1]. However, the properties of nuclei are clearly changing when shifting toward the isotopes that are far from the valley of stability. Our strategy is to investigate the fundamental interactions between the nuclear constituents via the collectivity in the spin-isospin channel as a function of the protons-neutron asymmetry.

The $(p, n)$ charge-exchange reactions at intermediate energies (200-300 MeV/u) are powerful tool to study the spin-isospin excitations of nuclei. The method of inverse kinematics $[2,3]$ enables us to study the $(p, n)$ reactions on exotic nuclei with high luminosity: in this technique neutron detectors are used for measuring the Time-of-Flight (ToF) of low-energy recoil neutrons in the kinetic energy range of $100 \mathrm{keV}-10 \mathrm{MeV}$. This technique was already applied to study the Gamow-Teller strength distribution from ${ }^{56} \mathrm{Ni}[3,4]$ and ${ }^{132} \mathrm{Sn}[5]$ isotopes. However, the cross sections of the charge-exchange reactions are very low, it is crucial to efficiently tag these reaction channels and minimize contaminant events from other reaction channels with larger cross-section and eliminate background due to gamma rays coming from the environment. For future experiments with higher beam intensities this background event will lead to higher trigger rate that cannot be handled with data acquisition systems (increasing the dead time).

One possibility to solve this problem is to tag the low-energy recoil neutrons in real-time, i.e. before recording the event data, by online neutron-gamma discrimination. The first generation of neutron detectors designed for these $(p, n)$ measurements, such as WINDS [6] at RIKEN RIBF, LENDA [7, 8] at NSCL/MSU or ELENS [9] at ATOMKI are incapable of distinguishing between neutrons and gamma rays. Our new device is a plastic scintillator based detector, sensitive to the differences between neutrons and gamma rays. This development will result a reduced trigger rate (by removing the background due to gamma rays), smaller data size and an additional parameter in the offline analysis.

Plastic scintillators with pulse shape discrimination (PSD) capability had been long sought after [10] until Zaitseva et al. [11] introduced a plastic scintillator with good PSD comparable to that of organic liquid scintillators. The neutron-gamma discrimination abilities of these EJ-299-33 and EJ-299-34 plastic scintillators have already been studied [12, 13, 14]. The shapes and sizes of the above counters, however, are rather limited to a conventional cylindrical shape with sizes of a $2.5-5 \mathrm{~cm}$ and they were with only one sided readout. Instead, we use a $30-\mathrm{cm}$ bar-type detector with readouts on both sides. In order to obtain a larger counter, we choose EJ-299-34 as the scintillator material. While its PSD properties are reported as slightly poorer [15] than those of EJ-299-33, it is harder plastic and therefore more easily machined and polished to high tolerance. 


\section{The prototype detector}

Our new device will consist of a plastic scintillator bars. The detector is designed to measure neutron energies using the $\mathrm{ToF}$ technique in the kinetic-energy region from a few ten $\mathrm{keV}$ to a few $\mathrm{MeV}$. A picture of the existing prototype bar is shown in Fig. 1.

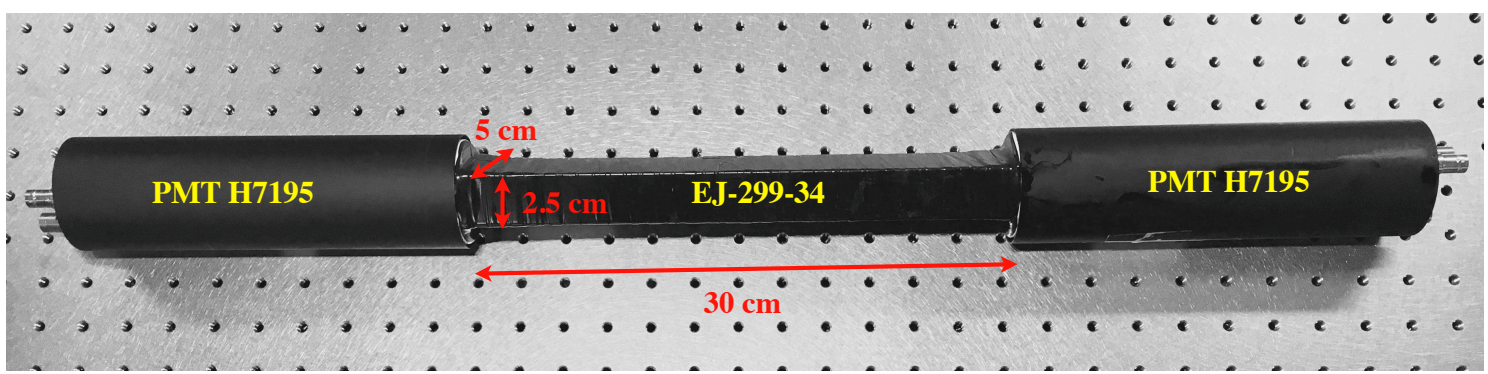

Figure 1: A picture of the prototype bar.

The bar-type prototype detector with dimensions of $2.5 \times 5 \times 30 \mathrm{~cm}^{3}$ is the largest existing sample of EJ-299-34 fast plastic scintillator [15], which can be produced by the Eljen Technology. The manufacturer quotes a light yield of 8600 photons $/ \mathrm{MeV}$ (at $1 \mathrm{MeV}_{e e}$ ) and an emission spectrum dominated by $420-425 \mathrm{~nm}$ wavelengths. The mean decay times of first 3 components of the light created in EJ-299-34 are 13 ns, 35 ns, 270 ns for gamma rays and 13 ns, 50 ns, 460 ns for neutrons.

The scintillator bar is wrapped with two layers of aluminized mylar foil, a layer of aluminum foil and finally black insulating tape for proper light propagation through the bar. The scintillator bar is coupled to a 51-mm-diameter-type Hamamatsu H7195 [16] photomultiplier tube (PMT) on each end. This PMT is selected due to its high photo-electron gain of $3 \times 10^{6}$ and low anode dark current. The PMTs are coupled to the scintillator by using EJ-500 colorless optical cement [17]. Using the time and optionally pulse-height information from the two PMTs [7], the timing of a neutron hit as well as the hit position along the longest side can be determined.

\section{Digital data acquisition system}

\subsection{Pulse processing for pulse shape discrimination}

Our new detector employs a digital data acquisition system. Signals from the anode output of both PMTs are read-out with a CAEN V1730 waveform digitizer (14-bit, 500 MSample/s flash ADC). The 2-ns sampling clock of the module is suitable for signals where the information critical for particle identification situated in the tail region. The digitizer communicates with the digital data acquisition system via A3818A optical link, while the Digital Pulse Processing for the Pulse Shape Discrimination (DPP-PSD) firmware [18] from CAEN is running on the digitizer.

For particle identification the system applies the charge comparison method [19,20] based on measurements of the integrated charge over two different time regions of the input signals. The 
Table 1: Optimized values of PSD parameters.

\begin{tabular}{cc}
\hline PSD Parameter & Optimum value \\
\hline Long gate & 250 clocks $=500 \mathrm{~ns}$ \\
Short gate & 24 clocks $=48 \mathrm{~ns}$ \\
Gate offset & 23 clocks $=46 \mathrm{~ns}$ \\
Baseline & 32 clocks $=64 \mathrm{~ns}$ \\
\hline
\end{tabular}

neutron-gamma discrimination is possible in real-time by the digital signal processing obtaining the pulse shape discrimination parameter as:

$\mathrm{PSD}=\frac{Q_{\text {Long }}-Q_{\text {Short }}}{Q_{\text {Long }}}$,

where $Q_{\text {Long }}$ is the integrated charge in the long gate, while $Q_{\text {Short }}$ is the integrated charge in a short gate at the beginning of the signal. The $Q_{\text {Long }}$ used as the full charge corresponding to the signal.

Our device is the first large volume detector which has double-ended readout and aims to provide PSD. Previous studies used cylinder shaped plastics with single-end readout. In order to maximize the PSD capability of the detector with double-ended readout, we use the mean PSD value coming from the two PMTs defined as:

$\mathrm{PSD}_{\text {mean }}=\frac{\mathrm{PSD}_{\text {left }}+\mathrm{PSD}_{\text {right }}}{2}$.

In order to optimize [21] the particle identification capability, the number of FADC samples used in the total signal integration (long gate), the number of samples in the integration of the first part of the signal (short gate), the parameter about the starting point of the integration prior the trigger is generated (gate offset) and the number of samples used in the dynamical evaluation of the baseline (baseline value) were tuned. All parameters were optimized taking into account the criteria for real-time operation, such as, the relation of the gates and the trigger signal or the gate offset $\leq$ short gate $\leq$ long gate criteria [22]. The optimized PSD parameters are shown in Table 1 .

\subsection{Triggering scheme}

In our digital data acquisition system, a new triggering scheme allows the system to operate at low energy threshold while reducing the gamma background and data rates. An event is saved only if the top and bottom photomultiplier tubes of one detector bar fire in coincidence and the event is identified as neutron based on the PSD of both sides. Figure 2 shows the working principle representation of the logic gates implemented on the FPGAs. The FPGAs generate the coincidence signals via the on-board logic filters using operating principles. This triggering scheme can be easily modified further also. 


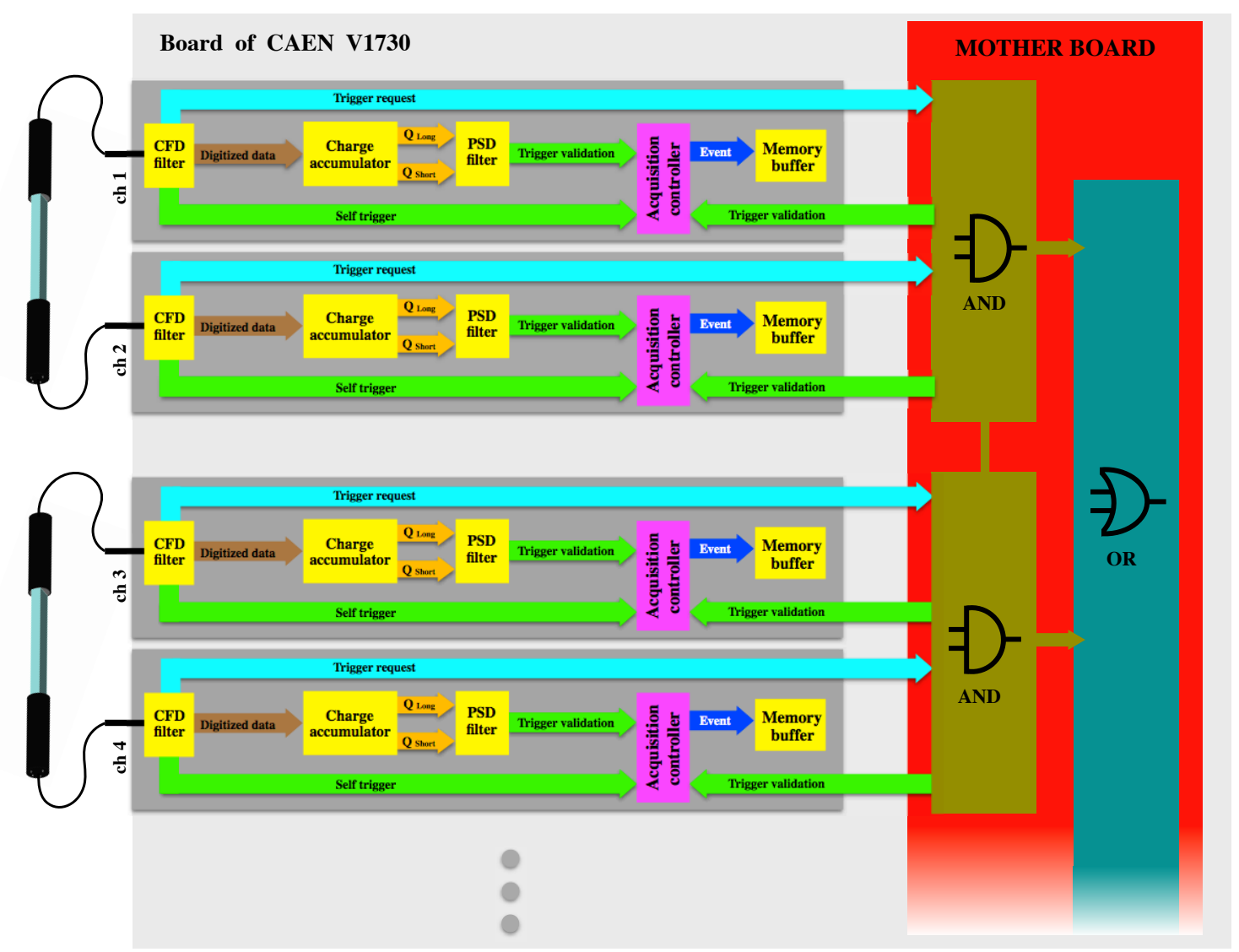

Figure 2: The working principle of the logic gates implemented on-board. The trigger validation comes separately from mother board and from PSD filter.

The triggering logic contains separated stages. At first stage, the board can trigger on each channel individually: once the input signal overcomes a threshold (in case of leading edge trigger), or it passes a digital constant fraction discrimination filter the further pulse processing based on Software Trigger source mode starts. In Constant Fraction Discriminator (CFD) mode, the input waveform is attenuated by a certain factor, then the signal is inverted and delayed by a fixed time; the two signals are summed producing the so-called CFD bipolar pulse. The zero crossing of the CFD signal is taken as the time trigger, this limits the minimum widths of the short gate. The delay and the attenuation parameter were optimized to be $2 \mathrm{~ns}$ and $25 \%$.

In the next two stages the triggering based on the programmed criteria by software command. In order to require the above mentioned coincidences between the top and bottom PMTs, the local triggering option [23] of two-two coupled channels (where coupled channels are $n$ and $n+1$ ) of V1730 digitizer is used (second stage). The local trigger validation comes from the mother board if the other channel inside the couple also fired. This results couple coincidence. Parallel to this step, the charge integration is evaluated by the charge accumulator and the PSD value is obtained in the PSD filter. In case the PSD value overcomes the threshold in both channels, the trigger validation successful, the event is saved. This triggering logic provides sensitivity for signals from low-energy 
neutrons. Several detector bars can be connected in chain.

\section{Test measurement}

We investigated the efficiency of the real-time neutron separation of the new detector bar using the ToF information. The test measurement setup is shown in Fig. 3.

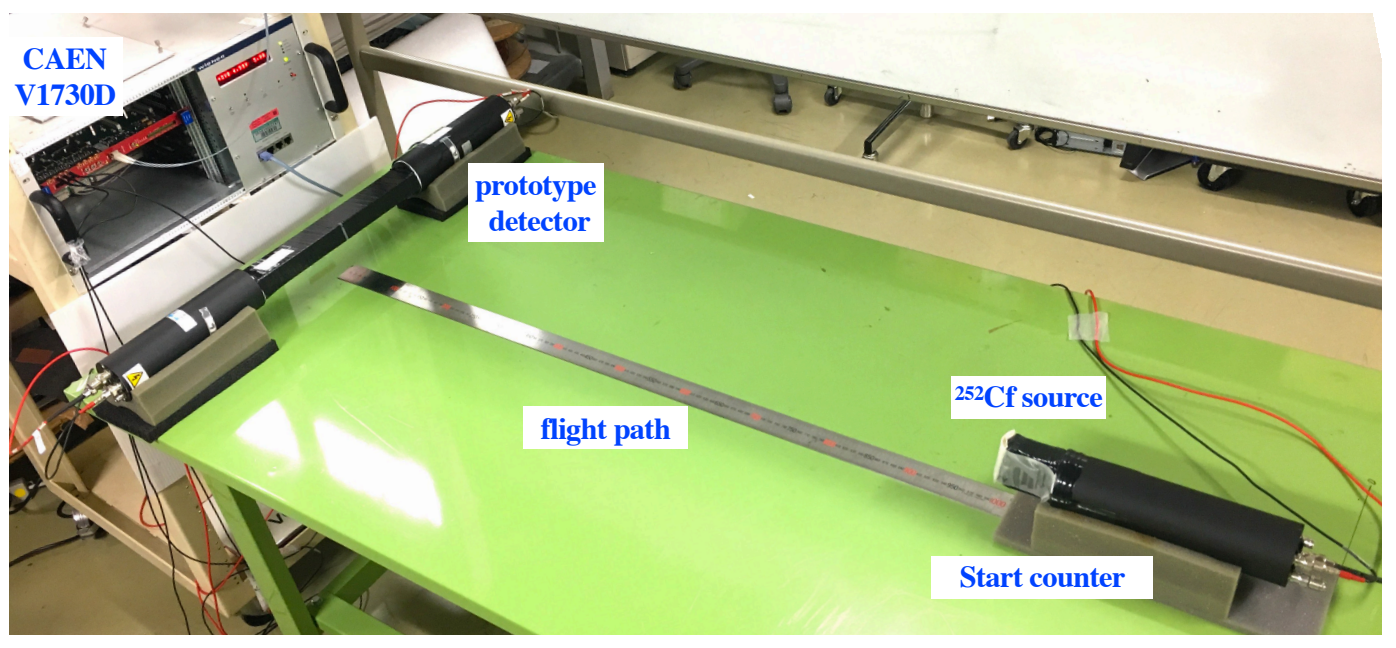

Figure 3: Photograph of experimental setup of ${ }^{252} \mathrm{Cf}$ source measurement. Prompt gamma-rays from fission were detected in a small plastic scintillator to generate the starting time of ToF measurement.

The ToF start signal was generated by the fission fragments detected using a small $(5 \times 5 \times 2.5$ $\mathrm{cm}^{3}$ ) plastic scintillator glued to the surface of a H7195 PMT. Gamma-rays are promptly emitted after the fission. The fission source was placed at a distance of $1 \mathrm{~m}$ from the neutron detector. The out-coming neutrons and gammas were not collimated by shadow bar or shielding and thus the total surface of the front face $\left(2.5 \times 30 \mathrm{~cm}^{2}\right)$ was irradiated. The voltage applied to the PMTs were 2150 $\mathrm{V}$, which was the optimized value. The light output responses were calibrated by ${ }^{241} \mathrm{Am},{ }^{137} \mathrm{Cs}$ and ${ }^{22} \mathrm{Na}$ gamma-ray sources using their photo peaks and Compton edges. The light output is derived as the geometric mean of the $\left(\mathrm{Q}_{\text {Long }}\right)$ charge values measured in two PMTs.

To manage the coincidence requirement between the start counter and our prototype detector, one additional coincidence condition (majority option) of three channels fired in a 250 ns wide time widow was applied on FPGAs. The CFD discrimination thresholds were set to be $1.2 \mathrm{mV}$ which equivalent to $10 \mathrm{keV}_{e e}$ light output. The typical self-trigger rate for single PMT was $10-12 \mathrm{kHz}$.

The scatter plot shown in Fig. 4 provides an efficient way to assess the online PSD against the offline separation by ToF for gamma-rays and neutrons. The events included in the sharp peak around $3 \mathrm{~ns}$ are due to gamma-ray coincidences between our prototype detector and the small start counter. The events that lie on the right side of the spectrum (larger than $20 \mathrm{~ns}$ ) correspond to neutron events. It is clear that only a small fraction of gamma rays are located in the PSD range of neutrons.

The efficiency of online neutron separation can be evaluated by setting a certain PSD threshold. Keeping the detection threshold at $10 \mathrm{keV}_{e e}$ and selecting a $\mathrm{PSD}_{\text {mean }}$ threshold at 0.15 the efficiency 


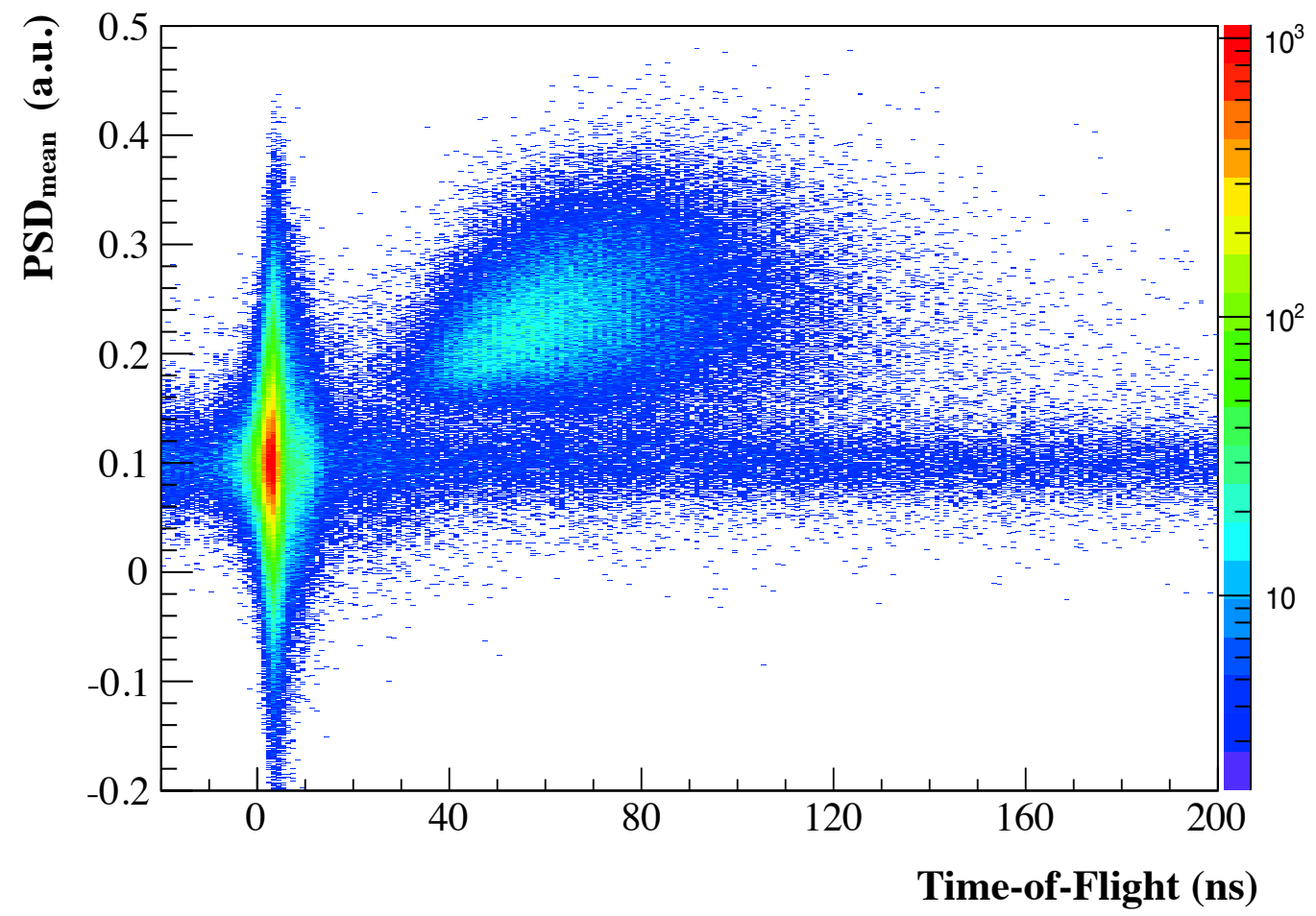

Figure 4: The $\mathrm{PSD}_{\text {mean }}$ vs. ToF spectrum shows a good separation of neutron- and gamma-like events. The sharp peak below 4 ns corresponds to events identified as gamma-rays, while the distribution in higher ToF region and above $0.15 \mathrm{PSD}_{\text {mean }}$ value represents the neutron-like events.

of the online neutron separation is more than $90 \%$ for all detected neutrons coming from the ${ }^{252} \mathrm{Cf}$ source. On the other hand, more than $90 \%$ of the detected gamma-rays can be excluded resulting in background reduction by one order of magnitude. In practical use the PSD threshold has to be tuned depending on neutron kinetic energy of interest and gamma-ray yield to be suppressed.

\section{Conclusions}

We have presented the first results on pulse shape discrimination capabilities of our new large volume plastic scintillator EJ-299-34 based device which is coupled to digital data acquisition system. The ToF distributions acquired using a ${ }^{252} \mathrm{Cf}$ fission source showed that more than $90 \%$ of all detected neutrons can be distinguished while more than $90 \%$ of the detected gamma-rays can be excluded. This online gamma rejection results in reducing the background by one order of magnitude. Our goal, the particle-based real-time triggering works properly. The device provides filtered data package of energy, timing, PSD information and digitized pulse shape. Our new setup provides opportunity for any reaction study that involves emission of low-energy neutrons.

\section{Acknowledgments}

This research was supported by The Japan Society for the Promotion of Science and Grant-inAid for Research Activity Start-up KAKENHI project 16H06716. It was also partially supported 
by Kurata Grant from the Kurata Memorial Hitachi Science and Technology Foundation.

\section{References}

[1] K. Nakayama, et al. Phys. Lett. B 114 (1982) 217.

[2] G. Perdikakis, et al., IEEE Transaction on Nucl. Science 56 (2009) 3.

[3] M. Sasano, et al., Phys. Rev. Lett. 107 (2011) 202501.

[4] M. Sasano, et al., Phys. Rev. C 86 (2012) 034324.

[5] PhD thesis of J. Yasuda, Study of Gamow-Teller transitions from ${ }^{132} \mathrm{Sn}$ via the $(p, n)$ reaction in inverse kinematics, Department of Physics, Kyushu University (2017).

[6] K. Yako, et al., RIKEN Accel. Prog. Rep. (2012) 45.

[7] G. Perdikakis, et al., Nucl. Instrum. Methods Phys. Res. A 686 (2012) 117.

[8] S. Lipschutz, et al., Nucl. Instrum. Methods Phys. Res. A 815 (2016) 1.

[9] L. Stuhl, et al., Nucl. Instrum. Methods Phys. Res. A 736 (2014) 1.

[10] J.B. Birks, The theory and practice of scintillation counting, in: International Series of Monographs on Electronics and Instrumentation, vol. 27, Pergamon Press, Oxford, 1964.

[11] N. Zaitseva, et al., Nucl. Instrum. Methods Phys. Res. A 668 (2012) 88.

[12] S.A. Pozzi, et al., Nucl. Instrum. Methods Phys. Res. A 723 (2013) 19.

[13] D. Cester, et al., Nucl. Instrum. Methods Phys. Res. A 735 (2014) 202.

[14] P. Blanc, et al., Nucl. Instrum. Methods Phys. Res. A 750 (2014) 1.

[15] EJ-299-33 and EJ-299-34 Plastics Datasheet: http://www.eljentechnology.com/products/plastic-scintillators/ej-299-33a-ej-299-34

[16] Hamamatsu H7195 Datasheet: https://www.hamamatsu.com/eu/en/product/alpha/P/3002/H7195/index.html

[17] EJ-500 Optical Cement Datasheet: http://www.eljentechnology.com/products/accessories/ej-500

[18] Digital Pulse Processing for the Pulse Shape Discrimination (DPP-PSD): http://www.caen.it/csite/CaenProd.jsp?parent=39\&idmod=770

[19] Y. Kaschuck, et al., Nucl. Instrum. Methods Phys. Res. A 551 (2005) 420.

[20] D. Wolski, et al., Nucl. Instrum. Methods Phys. Res. A 360 (1995) 584.

[21] J.H. Heltsley, et al., Nucl. Instrum. Methods Phys. Res. A 263 (1988) 441.

[22] CAEN User Manual: UM2580, Digital Pulse Processing for Pulse Shape Discrimination, Rev. 8 (2016).

[23] CAEN User Manual: UM4380, Registers Register Description for 725 and 730 DPP-PSD, Rev. 3 (2016). 